\title{
Synthetic Biology - a Key Driver of the BioEconomy through BioDesign and Industrial Translation
}

\author{
Richard I. Kitney \\ EPSRC National Centre for Synthetic Biology and Innovation (CSynBI) \\ UK National Industrial Translation Centre for Synthetic Biology (SynbiCITE) \\ Imperial College London \\ United Kingdom
}

Synthetic biology/engineering biology is highly disruptive technology that can be applied across a wide range of fields - well beyond the scope of traditional industrial biotechnology. It is seen as a key driver of the BioEconomy and is part of an alternative industrial model that is based on bio-based feedstocks and synthetic biology (rather than oil-based feedstocks and synthetic chemistry). In terms of productivity, synthetic biology/engineering biology represents three major gain factors: biology itself (that can often replace difficult, or even impossible, steps in industrial processes using chemistry); it naturally lends itself to the extensive application of automation (e.g. foundry technology), and AI. The seminar will discuss the translation of synthetic biology/engineering biology research from the laboratory to industry. Key elements of this process comprise standards, reproducibility and reliability. The basis of the synthetic biology/engineering biology consists of the application of engineering science. New technical standards for the field will be described and how these assist in the reproducibility of bio designs. Information systems are also an important element of bio design. A fully web-based information system will be described that allows the ability to characterise biological components in detail. A second important component in the process of reproducibility comprises the increasing use of DNA foundries. The elements of the Imperial foundry (the London DNA foundry) will be described and how this is part of an overall information infrastructure. Optimisation is an important aspect of BioDesign. This will be discussed in the context of a case study comprising the lycopene pathway and the use of DOE techniques. The final part seminar will address the next stages of industrial translation and how this relates to the agenda for the future of the Bioeconomy. 